\title{
Análise de adequabilidade dos indicadores de sustentabilidade urbana para avaliar áreas verdes e de recreação: Estudo em Vitória, ES 1
}

\section{Suitability analysis of urban sustainability indicators to evaluate green areas and recreation: Study in Vitória, ES}

\section{Lugão, Layra Ramos'; Bonatto, Daniella do Amaral Mello²; Alvarez, Cristina Engel de ${ }^{3}$}

\author{
1 Universidade Federal do Espírito Santo; Avenida Fernando Ferrari, 514, \\ Departamento de Arquitetura e Urbanismo, Vitória, Espírito Santo, Brasil; \\ layra.lugao@aluno.ufes.br \\ 2 Universidade Federal do Espírito Santo; daniella.bonatto@ufes.br \\ 3 Universidade Federal do Espírito Santo; cristina.engel@ufes.br
}

\begin{abstract}
RESUMO
A crescente urbanização mundial vem transformando o panorama geral das cidades em função da necessidade de abrigar novos moradores, muitas vezes ocasionando um crescimento desordenado e consequente desequilíbrio ambiental. Diferentemente de outras localidades do mundo, o contexto urbano dos países latino-americanos se caracteriza por sua fragilidade social e econômica, fazendo com que na avaliação de sustentabilidade não se possa ignorar a situação de contrastes em suas cidades. O objetivo deste artigo, parte de um estudo de mestrado, foi analisar a adequabilidade dos indicadores de sustentabilidade urbana da Iniciativa Cidades Emergentes e Sustentáveis do Banco Interamericano de Desenvolvimento para avaliar as áreas verdes e de recreação, em face da diversidade socioeconômica e desigualdades socioespaciais intraurbanas, através do estudo comparativo de duas regiões na cidade de Vitória (ES). Obteve-se como principal resultado a constatação de que as regiões carentes de áreas verdes e de recreação coincidem com os bairros de baixo índice socioeconômico. Conclui-se que a heterogeneidade da região deve ser considerada na análise da sustentabilidade, reforçando a complementação pela pesquisa de opinião dos moradores.
\end{abstract}

Palavras-chave: Sustentabilidade urbana, Indicadores de sustentabilidade, Metodologia de avaliação, Cidades emergentes e sustentáveis, Cidade de Vitória.

\footnotetext{
ABSTRACT

The increasing global urbanization has transformed the general panorama of the cities. Sheltering new residents as time goes by, a disorderly growth and consequent environmental imbalance is a reality. Unlikely others locations worldwide, the urban context of the Latin

${ }^{1}$ LUGÃO, Layra Ramos; BONATTO, Daniella do Amaral Mello; ALVAREZ, Cristina Engel de. Análise de adequabilidade dos indicadores de sustentabilidade urbana para avaliar áreas verdes e de recreação: Estudo em Vitória, ES. In: II SIMPÓSIO NACIONAL DE GESTÃO E ENGENHARIA URBANA: SINGEURB, 2019, São Paulo. Anais... Porto Alegre: ANTAC, 2019.
} 
American countries is characterized by its social and economic fragility. It is impossible to ignore the difficult situation of these cities in the assessment of sustainability. Part of a master's study, this article aimed to suitability analyze of the urban sustainability indicators of the Emerging and Sustainable Cities Initiative of the Inter-American Development Bank to evaluate green and recreational areas, in the face of socioeconomic diversity and intra-urban socio-spatial imbalances, through a comparative study of two regions in Vitória, Brazil. Therefore, it was suggested that regions lacking green areas and recreation coincide with low socioeconomic levels. Here, it is concluded that the heterogeneity of the region should be considered in the analysis of sustainability, reinforcing the complementation by opinion research with the residents.

Keywords: Urban sustainability, Sustainability indicators, Evaluation methodology, Emerging and sustainable cities, City of Vitória.

\section{INTRODUÇÃO}

O crescimento das cidades é uma das tendências globais mais significativas, que implica na complexa necessidade de recursos, infraestrutura e serviços nas cidades (BRAGANÇA et al., 2016). Segundo a ONU-HABITAT (2017), a população mundial tende a duplicar até 2050 e, consequentemente, as atividades econômicas, sociais e culturais a se concentrarem ainda mais nas cidades. A urbanização tornou-se uma das questões mais importantes no que diz respeito à relação do homem com a natureza, principalmente pelas alterações no meio ambiente natural e construído.

Um dos desafios para a sustentabilidade urbana é o de se tomar a paisagem como um todo e criar a interface necessária para um planejamento integrado - urbano e ambiental, visando a garantia da qualidade de vida (BONATTO, 2014). O papel do bairro é atender às necessidades sociais e ambientais através da variedade de uso do solo e tipos de edificações. No planejamento municipal, os espaços de parques e praças de bairro são negligenciados apesar da sua contribuição para a habitabilidade urbana devido à dificuldade de identificar e solucionar a insuficiência dessas áreas (FARR, 2013).

As ferramentas de avaliação da sustentabilidade urbana surgiram como mecanismo de suporte no reconhecimento das problemáticas e auxílio nas tomadas de decisões (BRAGANÇA et al., 2016). Sendo aplicadas em diversas escalas - local, nacional ou global muitas vezes sem analisar as particularidades de cada realidade, as ferramentas apontam soluções de contextos urbanos diferentes (YAN; WU; HUANG, 2015). Além dessa problemática, as áreas urbanas quase sempre se estendem além das fronteiras políticas ou administrativas, sendo que a avaliação da sustentabilidade somente para áreas definidas no perímetro urbano legal pode diminuir a interpretação do estado atual do ambiente se não compreendido com o entorno (FIALA, 2008, apud VERMA; RAGHUBANSHI, 2018).

Dentre as regiões com realidades urbanas semelhantes, as áreas urbanas da América Latina são caracterizadas pelos moradores estarem sujeitos à exclusão espacial, social e econômica tendo em consideração os amparos proporcionados pelo ambiente urbano (ONU-HABITAT, 2015). Para a transformação da realidade vigente, os indicadores devem destacar as potencialidades e fraquezas da área avaliada. Assim, o desenvolvimento sustentável contempla um conjunto de processos relacionados com as dimensões social, econômica e ambiental, sendo necessária uma avaliação equilibrada destes aspectos (LIMONAD, 2013).

Diante do exposto, o objetivo deste artigo foi analisar a adequabilidade dos indicadores de sustentabilidade urbana da Iniciativa Cidades Emergentes e Sustentáveis do Banco Interamericano de Desenvolvimento para avaliar as áreas verdes e de recreação, em face da diversidade socioeconômica e desigualdades socioespaciais intraurbanas, por meio do estudo comparativo de duas regiões na cidade de Vitória (ES) - Região de Maruípe e Região da Praia do Canto. 


\section{PROCEDIMENTOS METODOLÓGICOS}

O procedimento metodológico utilizado neste estudo consistiu em três etapas principais: i) análise dos indicadores de sustentabilidade urbana para espaços públicos da Iniciativa Cidades Emergentes e Sustentáveis do BID para duas regiões administrativas do município de Vitória-ES; ii) comparação dos indicadores com dados sociodemográficos e iii) análise da adequação dos referidos indicadores para se avaliar a sustentabilidade urbana de territórios desiguais, como é o caso de Vitória e outras cidades da América Latina e Caribe.

O estudo inicial acerca das ferramentas de avaliação existentes constata a tendência de avaliação para contextos semelhantes aos dos países de origem da ferramenta. Entretanto, apesar do país de origem ser caracterizado como desenvolvido, a ferramenta BID foi criada a partir da Iniciativa Cidades Emergentes e Sustentáveis (ICES) em resposta ao rápido processo de urbanização característico da região Latino-americana (BID, 2013). Com o total de 117 indicadores, a ferramenta BID possui uma proporcionalidade relativa em relação às dimensões social, econômica e ambiental (LUGÃO et al., 2018).

Em parceria com o BID, a Rede de Monitoramento Cidadão (RMC) sistematizou etapas de pesquisa que visam contribuir no acompanhamento do desempenho das cidades - incluindo a cidade de Vitória (ES) - em busca da sustentabilidade, e dentre estes, o Relatório de Análise de Progresso dos Indicadores (RAPI) e a Pesquisa de Opinião Pública (POP). Os relatórios foram resultados de levantamentos e 1069 entrevistas realizadas junto à população, por meio de um planejamento estrutural da metodologia e equipes responsáveis (BAOBÁ, 2017b).

Dentre os temas avaliados pela RMC, foi selecionado o tema "Espaços Públicos" - parques, praças e equipamentos urbanos - considerando tanto a sua importância no contexto da sustentabilidade como, também, a possibilidade de um estudo comparativo entre duas situações socioeconômicas diferenciadas.

No segundo momento, foram escolhidas duas regiões administrativas em Vitória que poderiam ser representativas da diversidade socioeconômica, sendo elas a Região Administrativa 4 - Maruípe; e a Região Administrativa 5 - Praia do Canto. Enquanto a primeira se caracteriza por sua população de baixa renda média mensal, a segunda por ser predominantemente de alta renda.

Assim, sendo o POP e o RAPI os resultados de uma das etapas da metodologia Cidades Emergentes e Sustentáveis, e a cidade de Vitória pertencente ao contexto de países Latinoamericanos, utilizou-se as informações levantadas da cidade alinhadas ao objetivo da pesquisa. Para o estudo comparativo, foram obtidos os dados e informações socioeconômicos junto à Prefeitura Municipal de Vitória (PMV, 2019) - área, bairros, população total, renda média e atividade econômica - e, posteriormente, avaliados em relação ao mapeamento socioeconômico e dos espaços públicos realizado pela RMC.

\section{RESULTADOS E DISCUSSÃO}

A partir da análise do referencial conceitual e estudos secundários, foi possível constatar as lacunas existentes entre a base teórica e as metodologias de avaliação de sustentabilidade, como por exemplo, a subjetividade na mensuração da influência social. Também observouse a grande quantidade de propostas de metodologias com uso de indicadores em contraposição à limitação da disponibilidade de dados para alimentar esses indicadores (VERMA; RAGHUBANSHI, 2018).

Dentre os indicadores-base propostos pela ICES relacionados ao tema "Espaços Públicos" foi escolhido para a análise comparativa, o subtema "Áreas verdes e de recreação", cujos indicadores são "Áreas verdes por 100.000 habitantes" e "Espaços públicos de recreação por 100.000 habitantes". Em um primeiro momento verificou-se a inexistência de dados disponíveis para o contexto analisado ou que não atendiam à metodologia de cálculo solicitada, como, por exemplo, as áreas verdes em hectares. Baobá (2017b) destaca ainda a importância do desenvolvimento deste subtema concomitante ao tema "Desigualdade Urbana".

A falta de dados para aplicação nos indicadores foi complementada com o resultado da 
Pesquisa de Opinião Pública (POP) realizada pela RMC para a cidade de Vitória (Baobá, 2017a), que aponta a percepção da sustentabilidade urbana pela visão dos moradores. Dentro do tema analisado, os moradores que dispõem de um espaço de lazer tendem a considerá-lo agradável e conservado. A preocupação com estes espaços se refere à segurança e ao número adequado para atender a população. Segundo Baobá (2017a), 17\% dos 1.069 entrevistados na cidade de Vitória afirmaram não haver espaços públicos de lazer em seus bairros, nas áreas identificadas na Figura 1.

Figura 1 - Mapeamento da existência de espaços públicos em Vitória

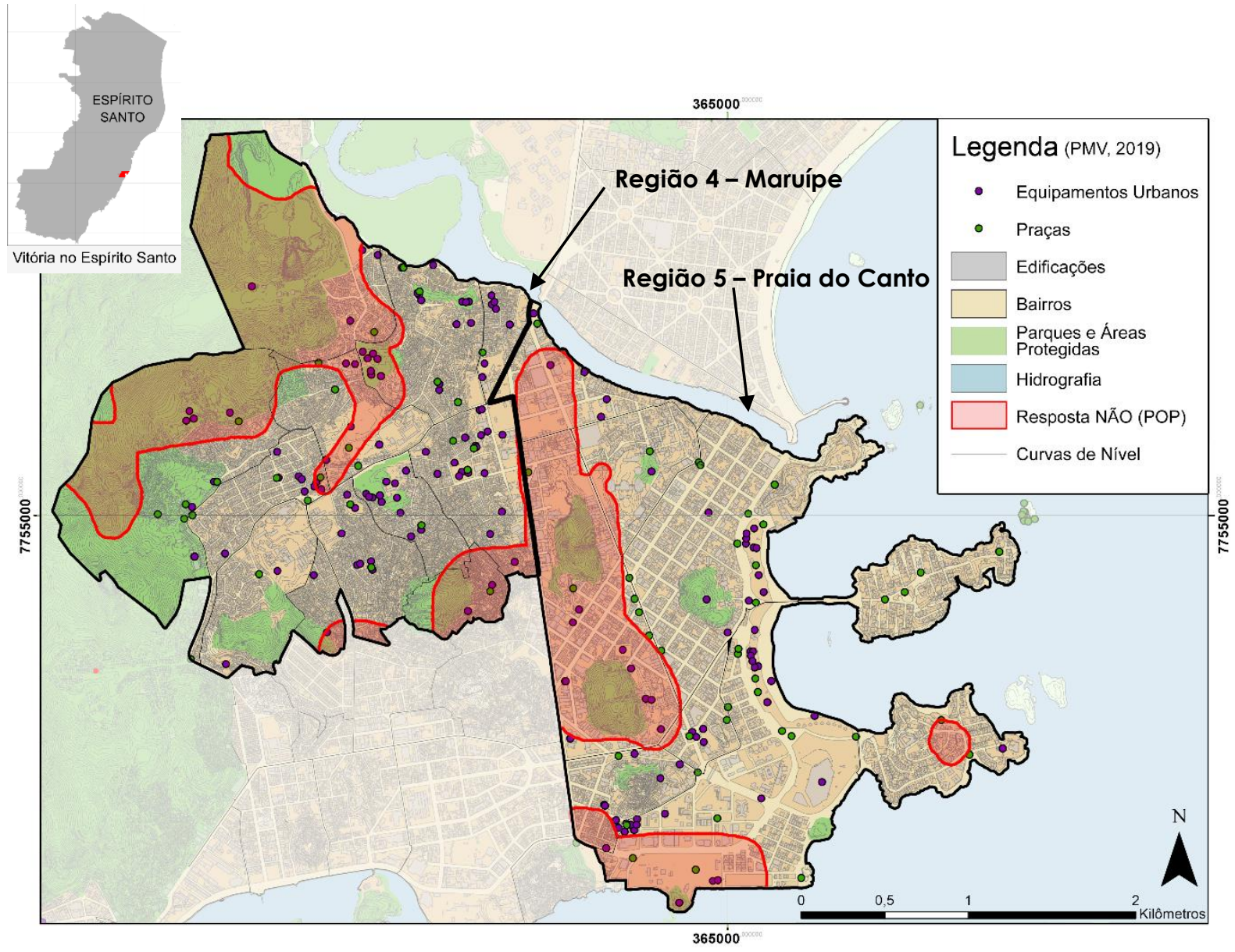

Fonte: Autoras (2019)

Como observado no mapa da Figura 1 e de acordo com Baobá (2017a), verifica-se a carência de espaços públicos nos bairros à noroeste de cada área administrativa. Além disso, segundo o levantamento da PMV (2019), a Região 4 possui 26 praças enquanto a Região 5 é contemplada com 42 praças.

Os dados coletados no POP fornecem um parecer da situação dentro dos limites da cidade. Localizadas próximas espacialmente, as Regiões Administrativas Maruípe e Praia do Canto da cidade de Vitória apresentam áreas equivalente com perceptíveis diferenças socioeconômicas. Na Tabela 1, o número total de habitantes é inversamente proporcional à renda média e atividade Econômica, em que computa os estabelecimentos da região que fomentam a economia local (PMV, 2019). 
Tabela 1 - Dados socioeconômicos das regiões administrativas

\begin{tabular}{l|c|c}
\hline & Região 4 - Maruípe & Região 5 - Praia do Canto \\
\hline Número de Bairros & 12 & 9 \\
\hline Área (Km²) & 5.684 & 5.334 \\
\hline População ( $\mathbf{n}^{\circ}$ habitantes) (2010) & 54.402 & 34.236 \\
\hline Renda média (2010) & $\mathrm{R} \$ 806,72$ & $\mathrm{R} \$ 3.844,97$ \\
\hline $\begin{array}{l}\text { Atividade Econômica ( }{ }^{\circ} \\
\text { estabelecimentos) } \mathbf{( 2 0 1 2 )}\end{array}$ & 10.903 & 48.150 \\
\hline
\end{tabular}

Fonte: Prefeitura de Vitória (2019)

As regiões selecionadas apresentam uma maior concentração populacional em áreas com menor renda média, enquanto a intensa atividade de comércio e serviços manifesta-se onde a renda média é maior. Na Figura 2 é possível verificar as diferenças de renda média mensal, sendo a da Região Administrativa Maruípe (4) identificada predominantemente com média de 1 a 3 salários mínimos, e a renda da Região Administrativa Praia do Canto (5) com variação de 4 salários mínimos a acima de 20. As delimitações das regiões coincidem com as dos bairros, porém verifica-se a transição gradual do contexto socioeconômico de uma para a outra.

Figura 2 - Renda mensal nas regiões administrativas segundo a Pesquisa de Opinião Pública

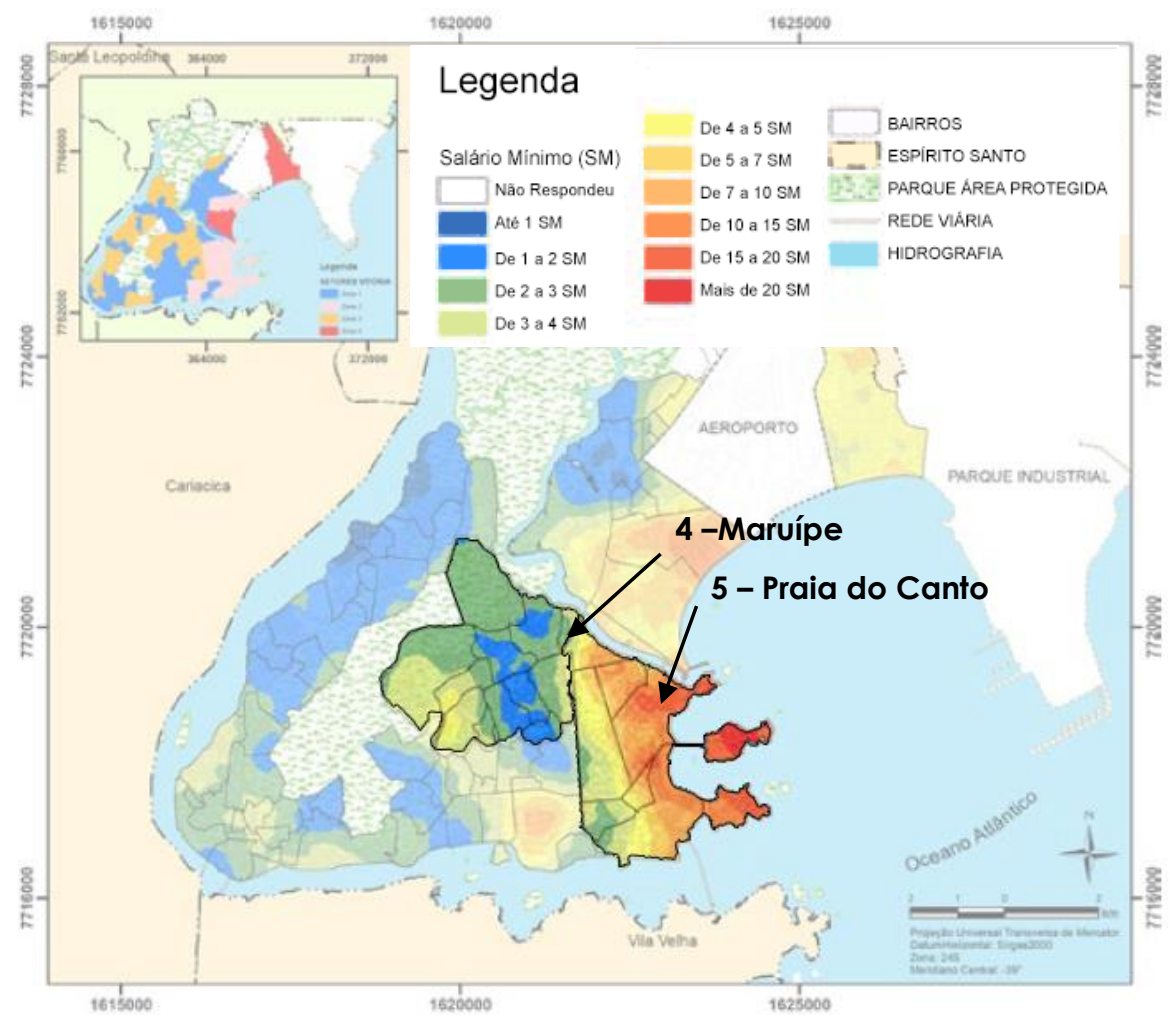

Fonte: BAOBÁ (2017a)

Comparando as Figuras 1 e 2, observa-se a prevalência da carência de espaços públicos nas áreas de menor renda mensal. Sendo a Região 4 mais populosa, seria necessário um maior número e diversidade de espaços públicos que atendessem àquela demanda, tanto em termos quantitativos como qualitativos. Outro ponto relevante a ser observado é a topografia local, visto que os bairros da Região 4 encontram-se em áreas de maior declividade e 
dificultam grandes áreas livres de lazer, em contraponto à Região 5, mais plana e com vasta orla de lazer (Figura 3).

Figura 3 - Topografia de Vitória

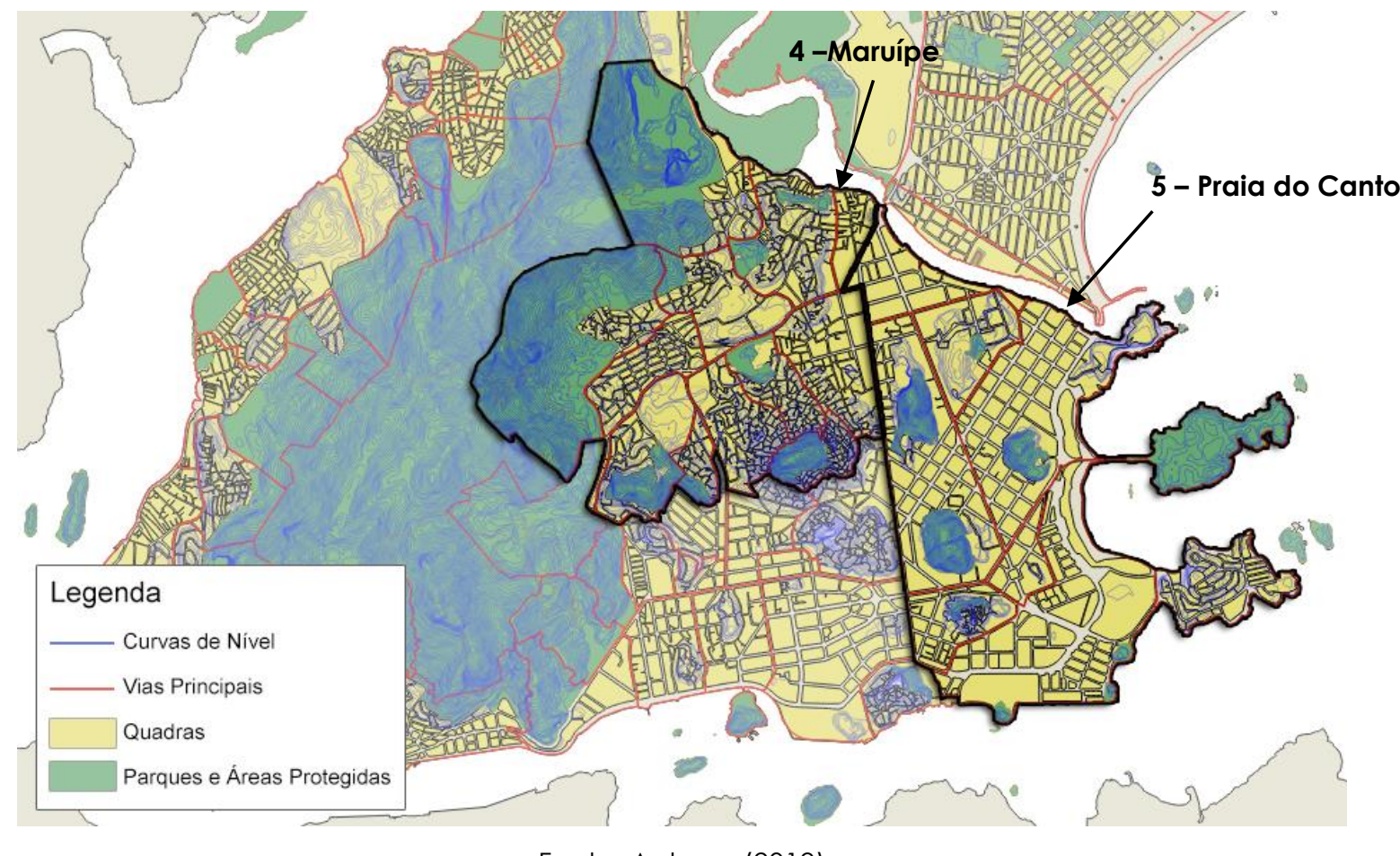

Fonte: Autoras (2019)

Em síntese, os indicadores de espaços públicos apontam a quantidade satisfatória de áreas verdes e espaços públicos por habitante considerando toda a cidade. Vitória tem 835,49 hectares de área verde por 100.000 habitantes estando acima do valor de referência ideal da ICES, de 50 hectares (OBSERVAVIX, 2017). No entanto, é perceptível a importância da análise intraurbana, visto que algumas regiões - como as exemplificadas neste estudo apesar da similaridade na área total, apresentam particularidades locais que interferem na quantidade de espaços públicos e na sua distribuição espacial. Tal observação fundamenta o entendimento de que indicadores que tratam apenas de médias municipais irão mascarar as desigualdades socioespaciais, que devem ser combatidas para que haja, de fato, maior sustentabilidade urbana.

\section{CONCLUSÕES}

Tendo em vista o aspecto socioeconômico como desafio nos países Latino-americanos para o alcance de um desenvolvimento sustentável, os indicadores devem captar as diferenças na urbanização com elementos que permitam a comparação entre diferentes localidades. Além dessa problemática, a escassez de dados disponíveis ou calculados, conforme a metodologia dos indicadores, indica a necessidade de aprimoramento da avaliação tradicional pela análise intraurbana - baseada na morfologia e na comparação dos dados socioeconômicos - que permite evidenciar as desigualdades intraurbanas e seu enfrentamento pela gestão urbana.

Assim, os indicadores como um instrumento para o planejamento urbano podem enfatizar os aspectos críticos considerando a disponibilidade de informações padronizadas, enquanto a opinião dos habitantes permite uma base qualitativa para a análise do contexto. Portanto, a cidade pode apresentar como resultado para um determinado indicador - como no exemplo relacionado aos espaços públicos - um valor que não condiz com a realidade ou necessidade do lugar, não sendo possível sua aplicação sem uma complementação da análise. 


\section{REFERÊNCIAS}

BAOBÁ. Práticas Sustentáveis et al. Pesquisa de Opinião Pública 2017 - Vitória. Vitória: 2017a. 63 p. Disponível em <

https://www.redeveracidade.com.br/adm/admin/controllers/Biblioteca/upload/53f0a4dee6 a70632b3dcd1900f676d76/POP_Vix_Ebook.pdf>. Acesso em: 17 Set 2018.

BAOBÁ. Práticas Sustentáveis et al. Relatório Anual de Indicadores 2017 - Vitória. Vitória: 2017b. 42 p. Disponível em

<https://www.redeveracidade.com.br/adm/admin/controllers/Biblioteca/upload/bad043e5 7b0068deeb3e8de579dff108/RAPI_Vix_ebook.pdf>. Acesso em: 17 Set 2018.

BID. Anexo 2. Indicadores da Iniciativa Cidades Emergentes e Sustentáveis: Guia Metodológico. Banco Interamericano de Desenvolvimento. 2013. Disponível em: <https://issuu.com/ciudadesemergentesysostenibles/docs/indicadores_ices_po>. Acesso em: 11 Set. 2018.

BONATTO, D. A. M. Corredores verdes: entre o planejamento urbano e o planejamento da paisagem. In: $12^{\circ}$. Encontro Nacional de Ensino de Paisagismo em Escolas de Arquitetura e Urbanismo - ENEPEA, 2014, Vitória - ES. Anais...Vitória: Universidade Federal do Espírito Santo UFES, 2014. v. 1, p. 340-347.

BRAGANÇA, L. et al. Avaliação do nível de sustentabilidade em comunidades urbanas energeticamente eficientes. In: Alvarez, C. E. de; Bragança, L. T. Comunidades urbanas energeticamente eficientes. Vitória: 2016. EDUFES, p.12-21.

FARR, Douglas. Urbanismo Sustentável: desenho urbano com a natureza. Porto Alegre: Bookman, 2013.

LIMONAD, Ester. A insustentável natureza da sustentabilidade: da ambientalização do planejamento às cidades sustentáveis. Cadernos Metrópole. São Paulo: 2013. v. 15, n. 29, p. 123-1 42. Disponível em: <https://revistas.pucsp.br/index.php/metropole/article/view/15819>. Acesso em: 14 Set 2018.

LUGÃO, L.R. et al. Comunidades urbanas latino-americanas: equilíbrio nas questões sociais, econômicas e ambientais para o desenvolvimento sustentável. In: 14 Jornada Urbenere e $2^{a}$ Jornada Cires, 2018, Vila Velha. Anais eletrônicos... Vila Velha: Universidade de Vila Velha UVV, 2018. Disponível em: <https://www.ciresurbenere.com/>. Acesso em: 14 Abr 2019.

OBSERVAVIX. Portal do Observatório de Indicadores da Cidade de Vitória. 2017. Disponível em: <http://observavix.vitoria.es.gov.br/>. Acesso em: 31 Mai 2019.

ONU-HABITAT. Nueva Agenda Urbana. Quito: 2017. Disponível em: <http://habitat3.org/wpcontent/uploads/NUA-Spanish.pdf>. Acesso em: 23 Ago 2018.

ONU-HABITAT. Documentos temáticos da Habitat III: assentamentos informais. Nova York: 2015. Disponível em: <http://habitat3.org/wp-content/uploads/22-AssentamentosInformais_final.pdf>. Acesso em: 30 Set 2018.

PREFEITURA DE VITÓRIA - PMV. Vitória em dados. Vitória: 2019. Disponível em: <http://legado.vitoria.es.gov.br/regionais/home.asp>. Acesso em: 26 Set 2018.

VERMA, P.; RAGHUBANSHI, A.S. Urban sustainability indicators: Challenges and opportunities. Ecological Indicators. 2018. Elsevier, v. 93, p. 282-291.

YAN, L.; WU, J.; HUANG, L. "Defining and measuring urban sustainability: a review of indicators". Landscape Ecology. New York: 2015. Springer, v. 30, n. 7, p. 1175-1193. 